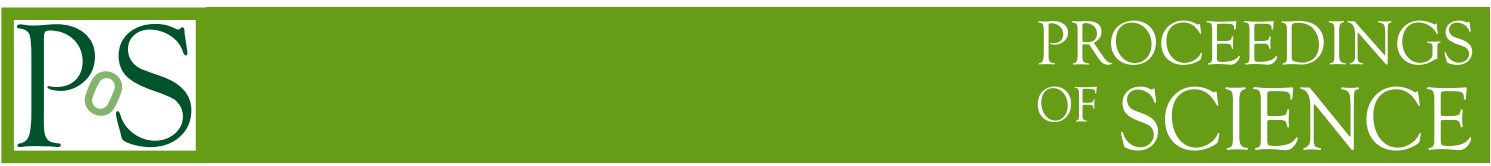

\title{
The AugerPrime Upgrade of the Pierre Auger Observatory
}

\author{
G. Cataldi ${ }^{a, *}$ for the Pierre Auger Collaboration ${ }^{b, 1}$ \\ ${ }^{a}$ INFN, Sezione di Lecce, via per Arnesano, 73100 Lecce, Italy \\ ${ }^{b}$ Observatorio Pierre Auger, Av. San Martín Norte 304, 5613 Malargüe, Argentina \\ E-mail: auger_spokespersons@fnal.gov
}

To answer many questions still open in the field of Ultra-High-Energy Cosmic Rays, the Pierre Auger Collaboration started a significant upgrade of the Observatory, called AugerPrime.

The main goal of the upgrade is to improve the mass composition sensitivity of the surface detector array on a shower-to-shower basis, in order to explore the cosmic ray composition at energies above $10^{19} \mathrm{eV}$. At energies unexplored by terrestrial accelerators, it will be possible to study the properties of multi-particle production and to search for new or unexpected changes of hadronic interactions. Moreover, in the region of the suppression of the cosmic ray flux, charged particle astronomy will benefit from the knowledge of the fraction of light primaries for composition-selected anisotropy searches.

After a discussion of the motivations for upgrading the Pierre Auger Observatory, a description of the detector upgrade is provided, together with an evaluation of the expected performance and the improved physics sensitivity. Finally the first data collected are presented.

40th International Conference on High Energy physics - ICHEP2020

July 28 - August 6, 2020

Prague, Czech Republic (virtual meeting)

${ }^{1}$ Full Author List: http://www . auger.org/archive/authors_2020_07.html

* Speaker 


\section{Introduction}

The Pierre Auger Observatory [1] is the world's largest cosmic ray experiment, designed for the indirect detection of the highest energy cosmic rays (namely above $10^{17} \mathrm{eV}$ ) through the detection of their atmospheric showers. It is a giant Observatory covering a surface of about $3000 \mathrm{~km}^{2}$ in a vast high plain in the Andes region of Pampa Amarilla (1400 m above sea level). The main components of the detectors are a surface array of 1660 ground stations and 24 fluorescence telescopes. Several enhancements extend the energy range of measurements in its lower part and provide different information, complementing the Surface array Detector (SD) and Fluorescence Detector (FD) data. The Observatory ("hybrid detector") employs two independent methods to detect and study highenergy cosmic rays. One technique detects high energy particles using ground arrays of surface detectors water-Cherenkov tanks (WCD), sampling the footprint of the air shower at ground level with $100 \%$ duty cycle. The other method tracks the development of air showers by observing the ultraviolet light emitted high in the Earth's atmosphere. This second technique can be applied only in clear, moonless nights and therefore it has a duty cycle of only 10-15\%. The unique exposure of the Observatory (over $60000 \mathrm{~km}^{2} \mathrm{sr}$ yr) acquired during more than a decade of data collection, has led to several steps forward in the field of Ultra-High-Energy Cosmic Rays (UHECR) physics. To name a few, the suppression in the energy spectrum above approximately $40 \mathrm{EeV}$ has been unambiguously confirmed to high precision [2] and a dipolar large scale anisotropy has been observed in cosmic rays with $\mathrm{E}>8 \mathrm{EeV}$ [3], proving that they are indeed of extragalactic origin and opening a window on the study of the properties of extragalactic magnetic fields. With these advances, however, Auger has also uncovered additional and unexpected complexity. One of the most remarkable is the general tendency towards heavier composition at the highest energies, which recalls questions concerning the origins of the flux suppression. The interpretation of the results in terms of composition is made more difficult by the large uncertainties in predicting hadronic multiparticle production at energies beyond those accessible in the laboratory. Moreover the studies aiming at the evaluation of the muon content in air showers [4] evidence inconsistencies in the modeling of hadronic interactions and muon production as implemented in the simulations. In order to extend the composition sensitivity of the Auger Observatory into the flux suppression region, an upgrade of the Auger Observatory (named AugerPrime [5, 6] ) has been planned. The main aim of AugerPrime is to provide additional measurements of composition-sensitive observables, allowing the determination of the primary mass of the highest energy cosmic rays on a shower-to-shower basis.

\section{The Detector Upgrade}

The upgrade consists of new plastic scintillator detectors (SSD) on top of the WCD of the surface array, an additional small photomultiplier (SPMT) installed in the WCD for the extension of the dynamic range, and new SD electronics [7]. Additionally an underground muon detector [8] is going to be deployed beside each WCD in the infill region of the Observatory, to provide direct muon measurements. The upgrade will also be complemented by extending the measurements of the existing FD [9] into periods of higher night-sky background, to increase their duty cycle. Finally, based on the Radio Engineering Array (AERA) experience and results, a new project for adding a radio detector to AugerPrime [10] is now on-going. 

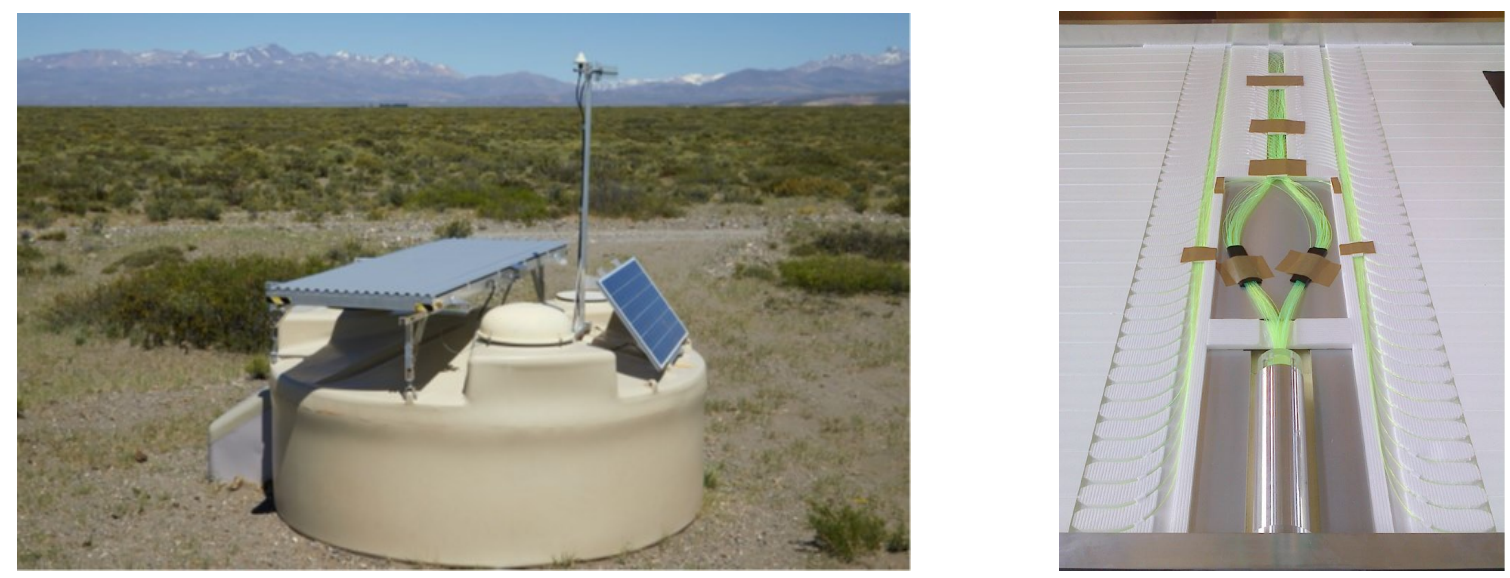

Figure 1: Left: One station of the AugerPrime Array, featuring the SSD on top of the WCD. Right: Picture of the inner part of a SSD detector with green wavelenght-shifting fibers routed from scintillators and guided in two bundles to the photomultiplier tube, housed in the aluminum support.

The key element of the upgrade is the SSD. A thin scintillation detector, which is mounted above and triggered by the larger WCD detector below it, provides a robust and well-understood way of particle detection that is sufficiently complementary to obtain a good measurement of the density of muons. In fact, complementing the WCD of the surface array with scintillator detectors will provide a way to sample shower particles with detectors having very different responses to the muonic and electromagnetic components. A SSD consists of a box of $3.8 \mathrm{~m} \times 1.3 \mathrm{~m}$, containing two scintillator sub-modules, each composed of extruded polystyrene scintillator bars of about $1.6 \mathrm{~m}$ length, $5 \mathrm{~cm}$ width and $1 \mathrm{~cm}$ thickness. The scintillator planes are protected by light-tight, weatherproof enclosures and are mounted on top of the existing WCD with a strong support frame, as shown in the left panel of Figure 1. The scintillator light is read out with wavelength-shifting fibres inserted into straight extruded holes in the scintillator planes, which are bundled and attached to a single photomultiplier tube, as shown in the right panel of Figure 1. The fibers are positioned following the grooves of the routers at both ends, in a " $U$ " configuration. They are bundled in a cookie, acting also as a diffuser, whose PMMA front window is connected to a single photomultiplier (PMT) tube. The assembly of the detectors has been distributed among different European sites of the collaboration, where the modules have also been singularly tested with cosmic-ray muons, in order to determine the detector response to a reference minimum-ionizing particle (MIP). The large-scale production of the SSDs started in 2017, and it is now completed. A considerable number of the detectors are being shipped in batches to Argentina where the local Argentinean team has the challenging task of transporting them to the different WCD stations often located in hard to reach areas. As of July 2020, over seven hundred detectors have been deployed, of which seventy-seven are operational. All the SSDs will be equipped with PMTs, and new electronics, as soon as these components become available. 

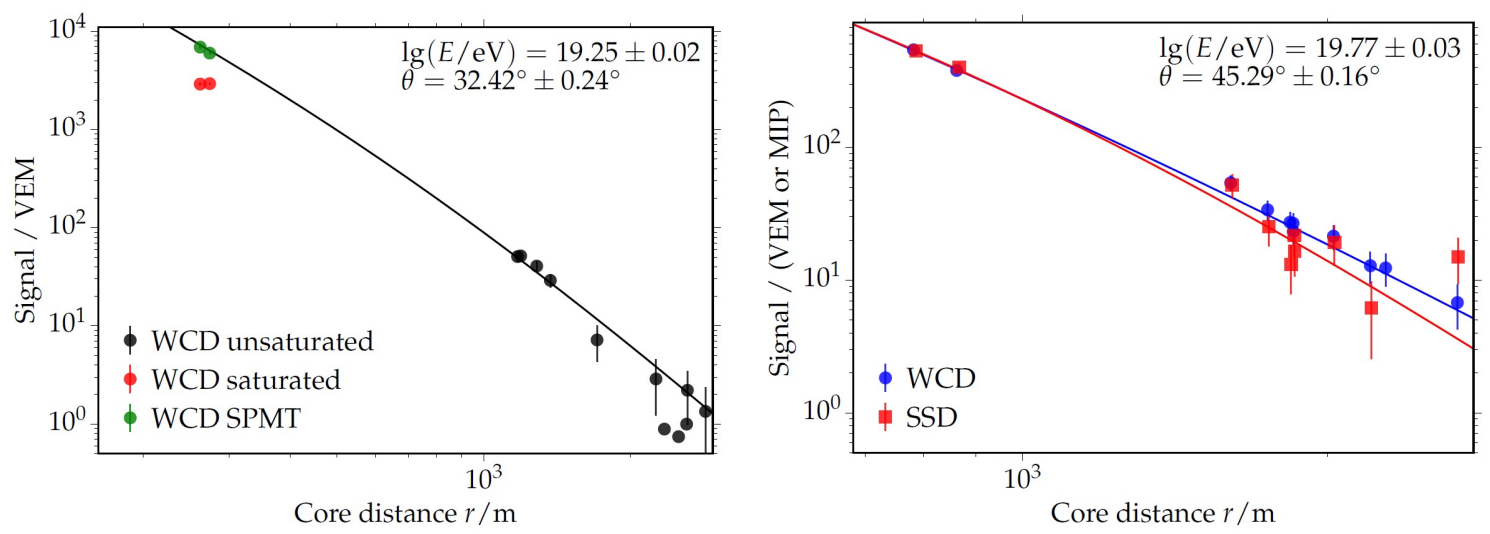

Figure 2: Left: Fit of the lateral distribution function for events with sPMT (event ID: 46663172). Right: Fit of the lateral distribution function of the WCD (blue) and the SSD (red) signals for a reconstructed event (ID: 53431866) with an energy of $60 \mathrm{EeV}$.

\section{Performance of the Upgraded Detectors}

Started in 2016, an Engineering Array of twelve AugerPrime stations has been operating in the field, with the aim of testing and monitoring the performance of the upgraded detectors. Additionally, seventy-seven SSDs have been deployed and are taking data since March 2019 (preproduction array). The SSD and WCD responses to shower components are very different. While electromagnetic particles have smaller energy deposits than muons in water, both components deposit on average the same amount of energy in the scintillator. In this sense, the SSD is more sensitive to the electromagnetic component of the shower.

After passing the event selection criteria, the standard shower reconstruction methods are applied to estimate the intrinsic properties of the primary cosmic ray. In the SD event reconstruction, the information about start times is used to reconstruct the arrival direction of the primary. After a first estimate of the arrival direction, a fit of the lateral distribution function (LDF) of signals at the ground is performed. This distribution arises from a convolution of the energy spectrum and the incoming direction of shower particles with the detector response. The example of an event reconstruction can be seen in the left panel of Figure 2. Signals from the WCDs are expressed in units of VEM (vertical-equivalent muon), which corresponds to the average signal resulting from a vertical and centered muon traversing the tank, measured as the integrated PMT pulse over time, thus a "charge". The term MIP (minimum ionizing particle) is used to express the signals from the SSD and it refers to the average charge deposited by a MIP. A sPMT inserted in the WCD allows the extension to measure the signal very close to the core of the shower where the standard PMTs of WCD evidence a saturation of the signal. The reconstructed lateral distribution function for one of the events collected in the region of the Engineering Array is shown in the right panel of Figure 2. The signal recorded in the upgraded WCD (blue dot) is in very good agreement with expectations, following the lateral distribution reconstructed by means of the standard stations. The SSD signal, in blue, is relatively lower than the WCD ones, as expected due to the different sensitivity of the two detectors to the electromagnetic component of the showers at different core distances and to the relatively smaller area of the SSD with respect to the WCD. The two detector types have different 

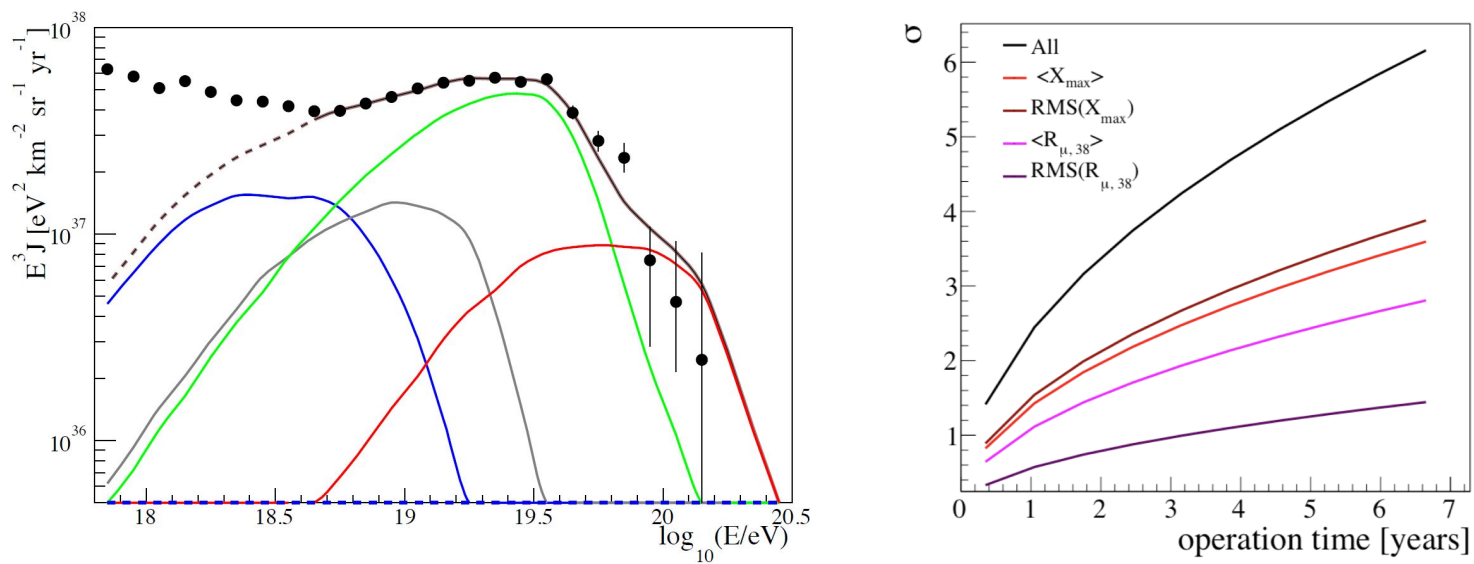

Figure 3: Left: Fluxes of different mass groups describing the Auger spectrum and composition data in the maximum-rigidity scenario. The colors are protons - blue, helium - gray, nitrogen - green, and iron - red. Right: Significance of distinguishing Scenarios $1 \mathrm{p}$ and 1 (with and without at least $10 \%$ of protons respectively) as a function of the operation time of AugerPrime (see text).

responses to the electromagnetic and muonic components of the air showers. Their signals are highly related to the electromagnetic energy flux and the muonic flux at ground. Therefore, signal densities sampled in the same position with WCD and SSD are essential for determination of the muonic shower component, which is crucial for primary particle identification.

\section{Science Impact of the Upgrade}

One of the key questions of the physics reach of the Auger Upgrade is that of being able to discriminate different composition and, hence, physics scenarios in the energy range of the flux suppression. This is very difficult to demonstrate without knowing what composition to expect. For this reason two benchmark simplified models have been chosen as representations of a maximumrigidity scenario (scenario 1) and one photo-disintegration scenario (scenario 2), as explained in detail in [11]. A fit to the Auger flux and composition data for $E>10^{18.7} \mathrm{eV}$ has been performed and the left panel of Figure 3 illustrates the fluxes of different mass groups in one of these scenarios. To explore the feasibility of detection of a small fraction of protons with AugerPrime in this scenario, a contribution of $10 \%$ of protons to it ("Scenario 1p") has been added. The difference between the means of the distributions as obtained from the two scenarios ("1" and "1p") divided by the statistical uncertainty is shown in the right panel of Figure 3 for several observables. As shown in the figure by the black line, the best separation power is obtained by constructing a combined significance using all available observables. We conclude that using this combination and integrating above $10 \mathrm{EeV}$ we might be able to distinguish between Scenario 1 and $1 \mathrm{p}$ (i.e. to observe a fraction of protons as low as $10 \%$ ) at the highest energies with more than $5 \sigma$ after five years of operation of AugerPrime. In addition to these studies, the availability of muon information on a shower-to-shower basis allows for many ways of studying features of hadronic interactions. For example, the correlation between the number of muons and the depth of shower maximum can be used to study general features of muon production, including the search for exotic interaction scenarios at very high energy. 


\section{Conclusions}

AugerPrime, the Pierre Auger Observatory upgrade, will provide a high statistics sample of events with mass information that will be able to measure the presence of even a small fraction of protons at the highest energies. An Engineering Array of twelve upgraded stations has been taking data in the field since 2016. In addition, seventy-seven SSDs were deployed and started data acquisition in March 2019 increasing the wealth of events at higher energies. The data collected so far demonstrate the quality of the new detectors and the physics potential of the upgrade project. The production and deployment of AugerPrime detectors and electronics is proceeding at full pace. Operations and full data taking are planned from 2021.

\section{References}

[1] A. Aab et al. [Pierre Auger Coll.],The Pierre Auger Cosmic Ray Observatory, Nucl. Instrum. Meth. A798, 172 (2015).

[2] A. Aab et al. [Pierre Auger Coll.], Features of the energy spectrum of cosmic rays above 2.5 x $10^{18} \mathrm{eV}$ using the Pierre Auger Observatory, Phys. Rev. Lett. 125, 121106 (2020).

[3] A. Aab et al. [Pierre Auger Coll.],Observation of a large-scale anisotropy in the arrival directions of cosmic rays above $8 \times 10^{18} \mathrm{eV}$, Science, 357, 1266 (2017).

[4] A. Aab et al. [Pierre Auger Coll.], Muons in air showers at the Pierre Auger Observatory: Mean number in highly inclined events, Phys.Rev. D91, 032003 (2015).

[5] A. Aab et al. [Pierre Auger Coll.], The Pierre Auger Observatory Upgrade "AugerPrime" Preliminary Design Report, in arXiv:1604.03637 [astro-ph.IM] (2015).

[6] A. Aab et al. [Pierre Auger Coll.], The Pierre Auger Observatory Upgrade , in proceedings of "35th International Cosmic Ray Conference" PoS(ICRC2017)383 (2017).

[7] A. Aab et al. [Pierre Auger Coll.], New electronics for the surface detectors of thePierre Auger Observatory, in proceedings of "35th International Cosmic Ray Conference" PoS(ICRC2017)450 (2017).

[8] A. Aab et al. [Pierre Auger Coll.], Prototype muon detectors for the AMIGA component of the Pierre Auger Observatory, Journal of Instrumentation, 11, P02012 (2016).

[9] J. Abraham et al. [Pierre Auger Coll.], The Fluorescence Detector of the Pierre Auger Observatory, Nucl. Instrum. Meth. A620, 227 (2010).

[10] A. Aab et al. [Pierre Auger Coll.], The Radio Detector upgrade of the Pierre AugerObservatory, in these proceedings (Contribution ID:950) (2020).

[11] A. Aab et al. [Pierre Auger Coll.], Combined fit of spectrum and composition data as measured by the Pierre Auger Observatory, in proceedings of "34th International Cosmic Ray Conference" PoS(ICRC2015)249 (2015). 